\title{
Correction to: Safety and efficacy of lamivudine or telbivudine started in early pregnancy for mothers with active chronic hepatitis B
}

\author{
Tianyu $\mathrm{He}^{1} \cdot$ Yuqing $\mathrm{Bai}^{3} \cdot \mathrm{Haodong} \mathrm{Cai}^{2} \cdot \mathrm{Xiaojuan} \mathrm{Ou}^{1} \cdot \mathrm{Min} \mathrm{Liu}^{3} \cdot \mathrm{Wei}^{\mathrm{Yi}^{3}} \cdot \mathrm{Jidong}^{\mathrm{Jia}}{ }^{1}$ (D)
}

Received: 31 May 2018 / Accepted: 31 May 2018/Published online: 11 June 2018

(c) Asian Pacific Association for the Study of the Liver 2018

\section{Correction to: Hepatology International (2018) 12:118-125 https://doi.org/10.1007/s12072-017-9839-5}

The aim of this erratum is to clearly state the individual author's contribution which was erroneously mentioned as "Tianyu He, Yuqing Bai, Wei Yi, and Jidong Jia contributed equally to this work."
The correct version is mentioned below:

Tianyu He and Yuqing Bai were co-first authors making an equal contribution; Wei Yi and Jidong Jia share cocorresponding authorship.

The original article can be found online at https:// doi.org/10.1007/s12072-017-9839-5.

\footnotetext{
Wei Yi

yiwei1215@163.com

$凶$ Jidong Jia

jia_JD@ccmu.edu.cn

Tianyu He

13439871617@163.com

Yuqing Bai

baiyuqingmd@163.com

1 Beijing Key Laboratory of Translational Medicine on Liver Cirrhosis, National Clinical Research Center of Digestive Diseases, Liver Research Center, Beijing Friendship Hospital, Capital Medical University, No. 95 Yong-an Road, Xicheng District, Beijing 100050, China

2 Department of Hepatology, Beijing Ditan Hospital, Capital Medical University, Beijing, China

3 Department of Obstetrics and Gynecology, Beijing Ditan Hospital, Capital Medical University, No. 8 Jing-shun East Street, Chaoyang District, Beijing 100015, China
} 